\title{
Smart Data Analytics in SME Manufacturing - an Axiomatic Design based Conceptual Framework
}

\author{
Andrew R. Vickery ${ }^{1, *}$,Erwin Rauch $^{2}$, Rafael A. Rojas ${ }^{2}$ and Christopher A. Brown ${ }^{1}$ \\ ${ }^{1}$ Worcester Polytechnic Institute, Department of Mechanical Engineering, 01609 Worcester, MA, USA \\ ${ }^{2}$ Free University of Bozen-Bolzano, Faculty of Science and Technology, Industrial Engineering and Automation (IEA), 39100 Bolzano, \\ Italy
}

\begin{abstract}
The objective of this paper is to go a step further introducing the concept of Industry 4.0 (I4.0) using Axiomatic Design as a research methodology. The rationale for this work is to increase the performance of SMEs allowing a better utilization of resources, using tools coming to the foreground of industry enabled by the Fourth Industrial Revolution (also known as Industry 4.0). This would improve the competitive position of SMEs on the global market. The state-of-the-art shows that only few companies and especially few small and medium sized enterprises (SMEs) are using data analytics to take advantage of new technological achievements to compete with one another. The method used in this paper for deriving a conceptual framework how SMEs can exploit the advantages of smart data analytics in manufacturing is axiomatic design, using inputs collected from a field study at SMEs. The results show that SMEs are challenged in implementing advanced data analytics instruments and need to define strategies for collecting, analysing and interpreting data for improving their performance and provide framework which should help SMEs to implement data analytics on the shop floor.
\end{abstract}

\section{Introduction}

Industry 4.0, or Smart Manufacturing, is currently revolutionizing production. After the first mention of Industry 4.0 at Hannover fair in 2011 and the proclamation of the fourth industrial revolution after mechanization, electrification and computerization, many large companies have already dealt with the topic [1]. In the meantime, many large companies $[2,3]$ as well as countries $[4,5]$ have started initiatives to introduce Industry 4.0 technologies or to incentivise companies to introduce them through government subsidies.

After initial scepticism, the research landscape in particular shows that the number of publications in the area of industry 4.0 has increased exponentially since 2016 (Scopus search with "industry 4.0": 91 hits in 2014; 213 hits in 2015; 527 hits in 2016; 1,090 hits in 2017; 2,045 hits in 2018) and that modern production can no longer be imagined without it. Large companies have the advantage of being able to provide enormous financial and human resources to drive the introduction of industry 4.0 concepts.

Small and medium-sized enterprises are very often disadvantaged here and have neither internal personnel skilled in the implementation of Industry 4.0 nor the money to set aside to conduct research. The fact that SMEs represent the backbone of many national economies [6] encourages the authors of this work to pursue targeted research for the introduction of industry 4.0 concepts in SMEs and to accompany them on this stony path.
Industry 4.0 very often mentions several enabling technologies. Some of these technologies are advanced manufacturing systems (e.g. collaborative robots), additive manufacturing, simulation and virtual/augmented reality, horizontal/vertical data integration, industrial internet, cloud computing, cyber security as well as big data and data analytics [7].

At the same time, a trend towards artificial intelligence [8], machine learning [9] and deep learning [10] can already be seen on the research horizon, which means that this aspect will be discussed even more in the coming years. In particular, topics such as data integration, connectivity and interoperability as well as big data analytics are currently still very difficult to implement for SMEs, although in many places there is a willingness to take the path of digitisation and to use digital instruments to make the company's processes more efficient [11].

This work wants to contribute in the research on data analytics for SMEs, as the initial situation in typical SMEs is reviewed on the basis of an as-is analysis by means of case study interviews in SME companies. Based on the interviews, the needs of the SMEs regarding data analytics are determined. Based on these needs Axiomatic Design is used as a method to derive design solutions how data analytics should be set up in SMEs. Finally, these design solutions are transformed into a framework, which will serve as a practical tool for SMEs to approach this introduction in a systematic and structured way. Furthermore, in a critical discussion at the end of this

\footnotetext{
*Corresponding author: andrewv@wpi.edu
} 
paper, necessary future research topics to enable data analytics in SMEs are identified.

\section{Theoretical Background}

This overview of the scientific literature focuses first the current status of the introduction of Industry 4.0 in SMEs. Subsequently, the current status of data analytics in SME manufacturing is highlighted. Based on the review the problem formulation for this paper is defined.

\subsection{Industry 4.0 in SMEs}

An analysis of scientific works and of projects to research the introduction of industry 4.0 in SMEs, conducted by the authors, shows, that the topic is still relatively "young" and unexplored as it has basically only been dealt with seriously since 2017 . Therefore, it will require further efforts to provide appropriate instruments for SMEs introducing Industry 4.0 in practice.

Analysing the content of 55 identified scientific papers in Scopus database (2013 - March 2019) with the search terms "Industry 4.0" and "SME" the following status can be summarized:

- Several works deal with innovative and digital business models to put SMEs in the position to take advantage of Industry 4.0 as a business tool.

- Researchers propose digital platforms to be interesting opportunities for SMEs to increase their business, but there are no relevant implementations of such SME platforms up to now.

- Several researchers are working on readiness, assessment or maturity models to help SMEs understand their true status.

- There is a rising number of works on frameworks, tool sets as well as roadmaps to guide SMEs in the implementation of Industry 4.0.

- It seems that Industry 4.0 is more and more interesting to other industries other than manufacturing (construction, textile, agriculture).

- Sustainability (in sense of ecological as well as social sustainability) is gaining attention.

- Although there is still little research about artificial intelligence and big data analytics in manufacturing in general, there must first be attempts to introduce low cost and easy approaches in SMEs.

\subsection{Data Analytics in SME Manufacturing}

Data analytics and derived data mining are powerful approaches for the analysis of big data. There are a lot of commercial data analytics applications that enterprises can take advantage of. Through efforts made in the field of the establishment of process standards, managers might be convinced of the advantages of Data Analytics. [12].

Despite the importance of small and medium-sized enterprises to all world economies, research in data analytics focuses mostly on large enterprises [13]. Many SMEs are still exempt from this development [12]. Dittert et al. [12] address the main reasons for SMEs' lack of attention to Industry 4.0, which are a lack of business prioritization, a lack of (IT) knowledge, and a lack of overview of data analytics issues.

The results of the literature review of Llave [13] show that general research on business intelligence (BI) and analytics increased substantially from 2011. Typical components of $\mathrm{BI}$ and data analytics applied in companies are $[13,14,15]$ :

- Identification and monitoring of key performance indicators (KPI),

- Data warehousing,

- Data mining,

- Online analytical processing (OLAP),

- Digital dashboards,

- Reports,

- Data visualization.

Reference models for enabling SMEs to overcome obstacles to introduce data analytics have been provided by Schütz et al. [16] or Schütz and Schrefl [17] which focuses on a reference model for data warehousing and the modelling of KPIs on an enterprise level. Another obstacle for SMEs working with data analytics is the need to extract knowledge from data warehouses in a way which allows navigation for non-expert users [13]. Several works are therefore dealing with easy-to-read dashboards and analysis tools [18, 19].

There are currently few works dealing with data analytics on the SME manufacturing shop floor. The authors in [20] report on the adoption of information technology in a large auto manufacturer, which has implemented agile manufacturing practices. The authors in [21] propose an architecture and system modules for big data analytics to implement smart factories in SMEs. The big data analytics platform enables SMEs to achieve an integrated system environment, to address quality issues by applying analytical models and to reduce their financial burdens through cloud computing. As an outcome this work suggests a manufacturing data analytics suite to provide consolidated information, including a data-mining model, its datasets, and preprocessing methods for specific manufacturing problems. The authors in [22] developed a concept of a masscustomizable data analytics service for manufacturing SMEs to support optimal decision-making in production systems. They also describe the problem that for many data analytics providers SMEs are not an interesting customer target group because of the low profitability for IT service providers. The authors in [23] suggest that although there may be some demand for big data analysis it is possible that the current solutions may not be viable for SMEs and that SMEs may be ill-prepared and illequipped to make the most of what big data analytics can offer them.

\subsection{Problem Formulation}

Based on the results of the literature analysis, the aim of this thesis is to derive a framework that supports practitioners in the introduction and installation of smart data analytics in SMEs. In addition, possible future fields of action for a successful introduction of data analytics in SMEs are to be identified. 


\section{Current Situation in SME Manufacturing: A Series of Case Studies}

In the following, the results of interviews with five case study companies from New England are presented to illustrate the status of production management and first steps to introduce I4.0 in SMEs.

\subsection{Company A: Sheet Rolling Machine Manufacturer}

This company is co-owned by two international conglomerates and makes best in class stock and sheet rolling machines for large industrial applications. The company has a strong top down hierarchical structure with the manufacturing floor being a job shop with categorical set up to machines.

Lean has been implemented to standardize processes. The company is moving to a new facility, dropping out of date machines, and acquiring new machines to increase standardization. Layout of the new facility will be optimized for current product lines.

This company has direct access to a local engineering university, giving it direct access to thought leaders. Leadership recognizes that I4.0 is needed in the future, shown by the creation of a position exclusively for the introduction of I4.0 in the machines it makes for its customers, though this is not being actively done for its own operating machines. The new facility is also being designed with I4.0 in mind, though what that means is still somewhat unrefined with regards to the infrastructure requirements for this to fully manifest.

The company has a mix of new and legacy equipment. The legacy machines have little to no sensor capability. Further, this company has a mix of new machines which have data pushing capabilities and legacy machines which do not. None of these machines talk in the same language, including different generations of the same machine and plans for the same part on different machines cost hundreds of man hours in re-programming in the new language for the new and old version of the same machine.

\subsection{Company B: Metal Components Manufacturer}

This company is family owned and manufactures the metal components to inserts for grinder machines which another company manufactures. They outsource the ceramic grinding surface and make the finally assembled insert on site. This company is largely paper run, with use of MS Excel spreadsheets for administrative work. Lean tools have been attempted with mixed success. The organizational set up is hierarchical.

This company has only legacy equipment and has been putting effort into creating retrofits to gain data from the machines. The company has one engineer and has limited access to a local university which it has a loose relationship with through the business school for operations education. The lack of a formal relationship with the local research university, a lack of dedicated I4.0 related efforts, legacy equipment, lack of budget, and being in a highly commoditized space make having the time, budget, and expertise for a concerted I4.0 effort difficult for this company.

\subsection{Company C: Plastic Washers Manufacturer}

This company is family owned and makes plastic washers for various industries and applications. The floor is organized as a job shop, with operations controlled through a series of MS Excel spreadsheets.

The company purchased a new plastic cutter for custom plastic sheet components which it is currently mobilizing in its product offerings.

This company has no engineers, no formal relationship with any university, its machines are legacy save for the one new machine. The company is in a commoditized industry and is aware it is behind the technological curve. It has no access to internal talent, funds, or time to begin an I4.0 initiative. The leadership believed the workforce was hostile toward I4.0 implementation. Its legacy machines mean they have no data upon which to build an information system about their operations.

\subsection{Company D: Badge Manufacturer}

This company is family owned, and makes badges, in recent years it has shifted from mostly a bulk manufacturer over to a boutique shop. They've begun making custom commemorative badges, and service badges in batches not large enough to send overseas. They consult on the design and aesthetics of the badges for the agencies they supply. The badge dies are made by a newly acquired desktop $\mathrm{CNC}$ machine, the only machine with any data capabilities. Each badge is forged in a legacy set of machines. Every badge design is different and requires hand colouring, creating scalability concerns. This company is largely hierarchical. The average age of someone in a leadership role is below 40 , the average age of a line worker is over 60 .

The integration of a CNC machine with a drafter making models for the badge designs has decreased labour around new badge designs. 3D printing for especially small orders is being looked at, with heavy push back from the line workers. One person in a leadership role was hesitant about the project purely from the fear that the workers would sabotage the machines.

The company has one engineer, working as a draftsman for CAD of new badge designs. The leadership is up to date on new industrial capabilities, and a loose relationship with a local university business school. The company has a hostile workforce against I4.0. The owners don't have impetus to act. Leadership has the desire, but no authority or internal talent to implement I4.0 technologies within the firm. The lack of standard work and lean practices means many hurdles for any modernization of operations.

\subsection{Company E: Polishing Machine Manufacturer}


This company is family owned and makes polishing machines for industrial applications. The company is organized as a job shop producing the polisher machines custom to order. A variety of machines for small to medium man operable machines, as well as robotic machines can be made.

All research and development go into improving the quality of the machines and the media with no attention being paid to the operations of making the machines.

This company has an active relationship with the surface metrology lab of a local university and has an engineer on staff. However, the company has no direct incentive to modernize operations. Though it makes I4.0 ready machines, its primary source of revenue is from selling media for the machines and aftermarket services for the machines they sell, meaning modernization of its machine manufacture may not have a high enough ROI from the viewpoint of leadership.

\subsection{Summary of the Case Study Analysis}

In summary, the situation in typical SMEs can be described as follows:

Due to the low personnel and financial resources, it is currently difficult for SMEs to introduce I4.0 on a large scale in the company. In addition, the scepticism of the employees often makes the efforts for innovations more difficult. To a large extent these enterprises are still managed on paper, whereby most employees are in the meantime familiar with handling simple and intuitive software applications and using also already mobile devices. A major difficulty is the fact that many of the existing machines are currently not ready to generate data or exchange information with higher-level systems. Despite all the difficulties, the management is willing to modernize the company and to start the digital transformation of their company, if a noticeable added value can be achieved.

\section{AD based design approach for smart data analytics in SME manufacturing}

As described in the problem formulation part this research wants to contribute to introduce I4.0 in SME manufacturing companies by focusing on the realization of simple data analytics suitable for smaller companies.

This section shows a procedure to obtain a first design concept and framework for smart data analytics in SME manufacturing. As a research methodology the research team used $\mathrm{AD}$ to derive a catalogue of design solutions. In the following subsections this procedure is described in detail.

\subsection{Axiomatic Design Methodology}

AD was developed by Nam P. Suh in the mid-1970s in the pursuit of developing a scientific, generalized, codified, and systematic procedure for design. $\mathrm{AD}$ uses the following four domains: 1) the customer domain where the customer wishes are described as so called customer needs (CNs); 2) the functional domain where $\mathrm{CNs}$ are translated into functional requirements (FRs) as well as design constraints (Cs); 3) the physical domain where design parameters (DPs) are derived that meet the previously defined functional requirements and 4) the process domain, where the DPs are transformed into real process variables (PVs).

The scientific theory gets its name from two axioms in $\mathrm{AD}$ that must be respected [24].

- The first is the Independence Axiom: Maintain the independence of the functional elements, i.e., avoid coupling in the system (e.g., avoiding dependencies between the DPs and other FRs).

- The second is the Information Axiom: Minimize the information content: select the solution with the least information content, i.e., that has the highest probability of success.

To apply these axioms, parallel functional and physical hierarchies are constructed, the latter containing the physical design solutions. The impact of AD is that the designer learns how to construct large design hierarchies quickly that are more structured, thus freeing more time for mastering applications [25].

In the initial workshop, requirements and so-called CAs were collected. Based on this input, FRs and Cs are defined and design parameters for a redesign were derived in an $\mathrm{AD}$ top-down decomposition and mapping process.

\subsection{Collection of Customer Needs}

Based on interviews and discussions with the companies described in section 3, nine common customer needs were gathered for the implementation of data analytics at their respective firms, as follows:

- $\quad C N_{l}$ : Final analytics information must be actionable for someone with little to no technical background.

- $\mathrm{CN}_{2}$ : Legacy equipment must be able to be integrated into final analytics system.

- $\quad C_{3}$ : Personnel must feel the analytics system is a value-added thing for them (not going to lose job, not going to have to do new arduous tasks or that they feel watched, etc).

- $\quad \mathrm{CN}_{4}$ : Be able to see the status of a product through production.

- $C N_{5}$ : Be able to see the status of machines (in use, not in use, down for maintenance, etc).

- $\quad C_{6}$ : Be able to project maintenance requirements and be able to mitigate the bottlenecks which would be caused by this.

- $\quad C N_{7}:$ Be able to see status of production capacity to have full knowledge of current orders, and capacity for future orders.

- $C N_{8}$ : Must enable real time visualization and communication of operations indicating information. 
- $\quad C N_{9}$ : Transformation of paper-based information into digital information accessible everywhere at any time.

These customer needs as discovered from the surveyed companies give a starting point for the decomposition to be discussed.

\subsection{Definition of Top-Level Functional Requirements and Design Parameters}

Based on the CNs previously identified the following $\mathrm{FR}_{0}$ and $\mathrm{DP}_{0}$ were defined:

$F R_{0} \quad$ Analyse and derive predictive decision making in SMEs

$D P_{0} \quad$ Framework for data analytics driven platform in SMES

Following, the research team defined the following top-level FRs and DPs.

\section{$F R_{1} \quad$ Determine data needs \\ $\mathrm{DP}_{1} \quad$ Data needs retrieval activities}

This upper level FR-DP pair indicates the requirement to determine who the need of standard and non-standard data could be defined. Further decomposition is required (see section 4.4.1).

\section{$\mathrm{FR}_{2} \quad$ Bridge needs for connectivity and interoperability \\ $\mathrm{DP}_{2} \quad$ SME Connectivity and Interoperability Hard-and Software}

This upper level FR-DP pair categorizes the hardware and software needs for connectivity and interoperability with and on the production shop floor. Further decomposition is required (see section 4.4.2).

\section{$\mathrm{FR}_{3} \quad$ Use available real-time data for prediction needs} $D_{3} \quad$ Data Analytics Tool Suite for SMEs

This upper level FR-DP pair ensures that real-time is used for further data analytics and that this will need specific and ease to use data analytics tools for SMEs. Further decomposition is required (see section 4.4.3).

\section{$\mathrm{FR}_{4} \quad$ Visualize and communicate information in an actionable format \\ $\mathrm{DP}_{4} \quad$ Visualization and Communication Tool Suite}

This upper level FR-DP pair fulfils the final deliverable of the tool suite, the presentation of actionable information to enable better decision making for people from production as well as at the enterprise level. Further decomposition is required (see section 4.4.4).

The interdependencies in the design matrix (see Eq. 1) show a decoupled design, meaning there is an established order of implementation of the design solutions for the adherence of the first axiom.

$$
\left\{\begin{array}{l}
F R_{1} \\
F R_{2} \\
F R_{3} \\
F R_{4}
\end{array}\right\}=\left[\begin{array}{cccc}
X & 0 & 0 & 0 \\
X & X & 0 & 0 \\
X & X & X & 0 \\
X & 0 & X & X
\end{array}\right]\left\{\begin{array}{l}
D P_{1} \\
D P_{2} \\
D P_{3} \\
D P_{4}
\end{array}\right\}
$$

\subsection{Decomposition and Mapping Process}

As mentioned before the top-level FR-DP pairs need to be further decomposed on a second level to get more tangible design solutions for the creation. The following subsections 4.4.1 to 4.4.4 show the results of the decomposition and mapping process and the related design matrices.

\subsubsection{FR $1-D P_{1}-$ Determination of data needs}

Based on the top-level FRs and DPs the research team derived the following lower level FR-DP pairs for determining data needs in SME companies.

$F R_{1.1}$ Determine data needs for production planning and control (PPC)

$F R_{1.2}$ Determine data needs for business management and controlling

$F R_{1.3}$ Determine individual and ad-hoc data needs

$D P_{1.1}$ Workshops on annual basis with production management

$D P_{1.2}$ Workshops on annual basis with top management and controlling

$D P_{1.3}$ Establishment of a contact person / position for individual data enquiries

To determine standard data needs that should be useful for production as well as for business management SMEs should organize once a year a meeting with production and a meeting with top management and controlling to define the key performance indicators (KPI) and data/information to be retrieved and analysed on a daily, weekly or monthly basis.

For individual, project-based or other ad-hoc data needs SMEs should define and establish a position with a skilled contact person to collect such data enquiries.

The design matrix shows an uncoupled design as all FRs are independent from each other.

$$
\left\{\begin{array}{l}
F R_{1.1} \\
F R_{1.2} \\
F R_{1.3}
\end{array}\right\}=\left[\begin{array}{ccc}
X & 0 & 0 \\
0 & X & 0 \\
0 & 0 & X
\end{array}\right]\left\{\begin{array}{l}
D P_{1.1} \\
D P_{1.2} \\
D P_{1.3}
\end{array}\right\}
$$

\subsection{2 $F R_{2}-D P_{2}-H W / S W$ for Connectivity and Interoperability}

Based on the top-level FRs and DPs the research team derived the following lower level FR-DP pairs for implementing necessary hardware (HW) and software (SW) for enabling connectivity and interoperability, which are indispensable for further data collection and analysis.

$F R_{2.1} \quad$ Enable data gathering of non-standard data 
$F_{2.2}$ Enable (vertical) data connectivity

$F_{2.3}$ Enable (horizontal) interoperability

$D_{2.1}$ Installation of specific sensors in production or on machines

$D P_{2.2}$ Vertical Data Integration

$D_{2.3} \quad$ Manufacturing Service Bus (MSB)

Based on the needs for data determined in $\mathrm{DP}_{1}$ SMEs must check if there is the need for installing specific sensors in production or on machines. Further a vertical connectivity must be achieved by Vertical Data Integration to allow the transfer of real-time information from the machine level to the manufacturing level and to the upper enterprise level [26]. In addition, for some data analytics tasks it may be necessary to exchange information on a horizontal machine level (between one machine and another). This is also called interoperability and can be achieved by installing a Manufacturing Service Bus [27]

The design matrix shows a decoupled design as the definition of specific sensors affects also the design and architecture of vertical data integration as well as the MSB.

$$
\left\{\begin{array}{l}
F R_{2.1} \\
F R_{2.2} \\
F R_{2.3}
\end{array}\right\}=\left[\begin{array}{ccc}
X & 0 & 0 \\
X & X & 0 \\
X & 0 & X
\end{array}\right]\left\{\begin{array}{l}
D P_{2.1} \\
D P_{2.2} \\
D P_{2.3}
\end{array}\right\}
$$

Vertical data integration is an important and not easy step for SMEs and therefore needs to be further decomposed on a third level to look for appropriate DPs.

$F R_{2.2 .1}$ Enable connectivity on machine level

$F R_{2.2 .2}$ Enable connectivity on manufacturing level

$F R_{2.2 .3}$ Enable connectivity on enterprise level

$D P_{2.2 .1}$ I4.0 ready machine interface

$D P_{2.2 .2}$ Manufacturing Execution System (MES) for SMEs

$D P_{2.2 .3}$ Enterprise Resources Planning System (ERP) for $S M E$

To enable vertical data integration, we need connectivity on several levels of the company. On the machine level the machines must be able to communicate with upper level systems, which means that machines must be equipped with I4.0 ready machine interfaces [28]. On the manufacturing level so called Manufacturing Execution Systems are currently being introduced that should close the gap of connectivity between the machine level and the upper enterprise level [29] where ERP systems are already implemented in most industrial companies and SMEs.

The design matrix shows a decoupled design as the integration of IoT Gateways and I4.0 interfaces on the machine level affect the design of interfaces with the MES system.

$$
\left\{\begin{array}{l}
F R_{2.2 .1} \\
F R_{2.2 .2} \\
F R_{2.2 .3}
\end{array}\right\}=\left[\begin{array}{ccc}
X & 0 & 0 \\
X & X & 0 \\
0 & 0 & X
\end{array}\right]\left\{\begin{array}{l}
D P_{2.2 .1} \\
D P_{2.2 .2} \\
D P_{2.2 .3}
\end{array}\right\}
$$

I4.0 ready machines are rare in SMEs, with only newer machinery being equipped with communication technology able to exchange data with other MES or ERP system. Therefore, we need to further decompose $\mathrm{DP}_{2.2 .1}$.

FR 2.2.2.1 Enable connectivity of new machines

$F R_{2.2 .2 .2}$ Enable connectivity of old machines

$D P_{\text {2.2.2.1 Integrate }}$ I4.0 readiness specification in purchasing requests

$D_{2.2 .2 .2}$ I4.0 Retrofitting through commercially available IoT Gateways

While for new machinery it is enough to integrate I4.0 readiness specifications in the purchasing decisions, the retrofit of old machines is more difficult. In the meantime, several devices are available on the market to update legacy machines and to enable them to communicate with other machines or software systems in sense of Internet of Things (IoT) capability. Some examples are the I4.0 box developed by [30] or the IoT Gateway of Bosch [31].

The design matrix shows an uncoupled design.

$$
\left\{\begin{array}{l}
F R_{2.2 .2 .1} \\
F R_{2.2 .2 .2}
\end{array}\right\}=\left[\begin{array}{ll}
X & 0 \\
0 & X
\end{array}\right]\left\{\begin{array}{l}
D P_{2.2 .2 .1} \\
D P_{2.2 .2 .2}
\end{array}\right\}
$$

\subsection{3 $\mathrm{FR}_{3}-\mathrm{DP}_{3}$ - Data Analytics Tool Suite}

The main functionality of the data analytics framework for SME companies is a data analytics tool suite. The following decomposition of $\mathrm{DP}_{3}$ shows the lower level FR-DP pairs.

$F R_{3.1}$ Gather real-time data from production

$F R_{3.2}$ Clear and pre-process unstructured data

$F R_{3.3}$ Analyse structured data

$F_{3.4}$ Interpret non-standard mined results/patterns

$D P_{3.1}$ Real-time data collection by using available data sources

$D P_{3.2}$ Automated data structuring and pre-processing

$D_{3.3}$ Selection and application of Data Mining approaches

$D_{3.4}$ Establishment of a position of production data analyst

According to Dittert et al. [12] and Llave [13] such a data analytics tool needs in a first step to retrieve realtime data from different sources like machines, sensors, MES, ERP, Customer Relationship Management (CMR) software or other sources like excel spreadsheets etc. In a second step this unstructured data needs to be cleared, preprocessed (removing features that are not necessary, detect and handle noisy or missing data, etc.). As soon as structured data are available this data needs to be analysed by selecting and applying the right approach for Data Mining. While standard results can be provided directly via predefined ways for communication and visualization (see DP4) non-standard data need to be interpreted by a skilled person. Therefore, also SMEs will need to qualify and install people as production data analysts. 
The design matrix shows a decoupled design as the results of pre-processing the data affects also the step of analysis of the data itself.

$$
\left\{\begin{array}{l}
F R_{3.1} \\
F R_{3.2} \\
F R_{3.3} \\
F R_{3.4}
\end{array}\right\}=\left[\begin{array}{cccc}
X & 0 & 0 & 0 \\
0 & X & 0 & 0 \\
0 & X & X & 0 \\
0 & 0 & 0 & X
\end{array}\right]\left\{\begin{array}{l}
D P_{3.1} \\
D P_{3.2} \\
D P_{3.3} \\
D P_{3.4}
\end{array}\right\}
$$

\subsection{4 $\mathrm{FR}_{4}-\mathrm{DP}_{4}-$ Visualization and Communication}

The main functionality of the data analytics framework for SME companies is a data analytics tool suite. The following decomposition of $\mathrm{DP}_{3}$ shows the lower level FR-DP pairs.

$F_{4.1} \quad$ Communicate data-based actions needed

$F R_{4.2}$ Visualize production relevant standard data

$F R_{4.3} \quad$ Visualize enterprise related standard data

$F_{4.4}$ Visualize non-standard data

$D P_{4.1}$ Installation of an Alert Function if limit values are exceeded

$D P_{4.2}$ Responsive digital production dashboard

$D P_{4.3}$ Responsive digital dashboard for controlling and top-management

$D_{4.4}$ GUI for individual data enquiries and visualization of results

If the results of data analytics detect an exceedance of predefined limit values of KPIs this should lead to an immediate alert. Standard production relevant data should be visualized on production dashboards realized in a responsive technology to visualize them on different mobile devices in and outside of the production shop floor (e.g. smartphone). The same occurs for data relevant for the top-management or for enterprise controlling. For individual data enquiries there should be implement also a graphical user interface (GUI) were interested people can send the enquiry and get the visualization of the results.

Figure 1 shows the comprehensive decomposition in a FR-DP tree.

The design matrix shows a decoupled design as the installation of alert functions affects also the design of dashboards in production and for top-management and controlling (integrating the visualization of such alerts on the dashboards).

$$
\left\{\begin{array}{l}
F R_{4.1} \\
F R_{4.2} \\
F R_{4.3} \\
F R_{4.4}
\end{array}\right\}=\left[\begin{array}{cccc}
X & 0 & 0 & 0 \\
X & X & 0 & 0 \\
X & 0 & X & 0 \\
0 & 0 & 0 & X
\end{array}\right]\left\{\begin{array}{l}
D P_{4.1} \\
D P_{4.2} \\
D P_{4.3} \\
D P_{4.4}
\end{array}\right\}
$$

\section{Framework for implementing Smart Data Analytics in SME Manufacturing}

The framework discussed lays out a procedure by which SMEs can implement data analytics for the prediction of various business processes to facilitate better strategic and tactical decision making for higher ROI on the SME's processes. The implementation of this framework illustrated in Figure 2 would go as follows.

The SME leadership would determine what processes need to have analytics implemented. This could be any scale or process within the SME, from one product line, to implementation in all business processes.

Once the scope of implementation is selected, the SME now needs to determine the data needs and resource requirements for PPC, management and controlling, and individual and ad-hoc data implementation. This is done through an ongoing series of workshops and assigned personnel to enable the ongoing development of the understanding of the data needs for the analytics tools which the SME needs.

Once the data and related resources are determined, gathering, connectivity, and interoperability of the desired data. This is enabled through an I4.0 ready machine interface which enables data aggregation systems such as an MES at the operational level and an ERP at the enterprise level. Interoperability between these different levels is enabled through an MSB.

Now that the data can move, it must now be gathered to a single collection point, structured, pre-processed, analysed, and interpreted.

Finally, this allows the visualization and communication of alerts, digital production dashboard responses, control and top-management level information, and the population of a GUI for individual data enquiries and visualization of results.

All of this allows for better understanding of not just where the SME is operationally and strategically, but potentially gaining insights into how strategic and tactical decisions within the SME could affect its future, potentially increasing the SME's ROI on various processes, and decrease variability within its processes. 


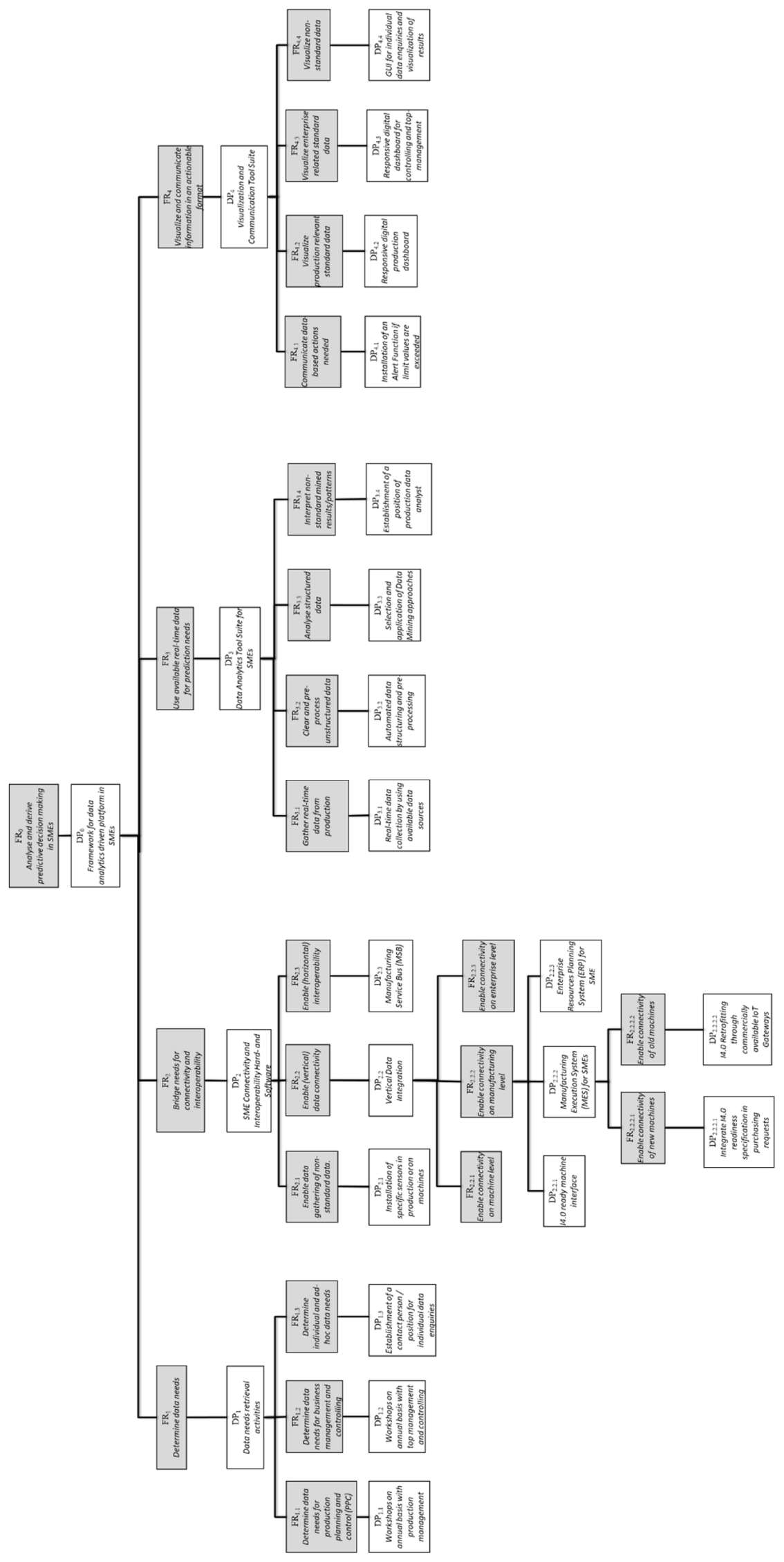

Fig. 1. Decomposition FR-DP tree.

\footnotetext{
* Corresponding author: andrewv@,wpi.edu
} 


\section{Discussion}

The final analytics information being actionable to someone with little technical background will be dependent on the usability of the GUI behind the visualization and communications tools. With the extensive sorting, collating, and refining put into the data, this will likely be a technology fluency problem rather than one where the user needs a high qualification in data science. The projection of maintenance requirements to manage possible bottlenecks is also a matter of the GUI for the final analytics tool suite should the hardware and data needs be properly implemented, as well as the ability of data analyst positions throughout the framework.

Legacy equipment is addressed for its hardware, and connectivity concerns within the framework. Real time data on the process and machines ensures status of products through production and machine status can be addressed through the final GUI of the analytics tool with proper visualization.

Real time visualization and communication of operations leading information, as well as transformation from a paper-based to digital information-based operation is addressed throughout the visualization and communication branches of the framework.

Though many of the CNs are well addressed, at least on a conceptual level. However, $\mathrm{CN}_{3}$ (value adding feeling for users) is not well addressed within the existing framework. Though the workers of the SME would have access help in development of the analytics implementation, through workshops, and contact personnel, and leadership communication, the workers of the SME could be considered a secondary customer to the leadership of the business. This must be considered as the framework is further fleshed out and implemented in future research.

Another gap in the framework is the addressing of the needs of SMEs with too little revenue to hire a team of data analysts to implement, curate, and maintain the accuracy of the outputs for the data analytics framework. This must be addressed in future works, but it may require a smaller, perhaps fully cloud based software platform which would allow the smallest of SMEs to integrate pieces of their operation into the framework as time, budget, and fluency with the framework allow.
Hardware needs, and data needs must be more fully explored with either a set of rules to follow to determine these needs, or a handbook with implementation guidelines. E.g. the development and commercialization as well as implementation of IoT gateways to make legacy equipment I4.0 ready.

Data needs for PPC, business management and controlling, and individual data need to be fleshed out further to a standard set of rules to start from for SMEs with little access to data scientists to still be able to utilize the tools which this research aims to be accessible to them.

The biggest gap in research will be the development of the Data Analytics Tool Suite itself as this is needs to be adapted to the needs and much more important to the financial capabilities and the qualification level of operators and production management staff in smaller manufacturing enterprises. Therefore, future research should address this challenge and contribute to develop, exploit and commercialize such tools.

\section{Conclusion}

The aim of this paper is to show SMEs how to introduce smart data analytics in their company and to identify what is necessary for this. To this end, interviews were conducted in various SMEs in New England to determine the SME requirements. These were finally transformed into a conceptual framework in the form of FRs and DPs using $\mathrm{AD}$ decomposition and mapping.

The contribution of this paper for practitioners is to divide a very complex problem, namely the introduction of smart data analytics in SMEs, into manageable small packages, to analyse their connections and to discuss the current possibilities and still open fields of action. For researchers this framework gives much scope for further investigation of the individual elements of the framework, in which the authors identified further need for research. Based on this work a first step for the development of data analytics tools is to be laid, which are easy to use and affordable also for SMEs.

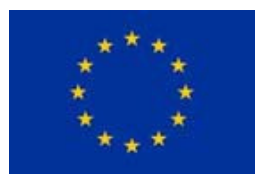

This project has received funding from the European Union's Horizon 2020 research and innovation program under the Marie Skłodowska-Curie grant agreement No 734713 .

\footnotetext{
* Corresponding author: andrewv@,wpi.edu
} 


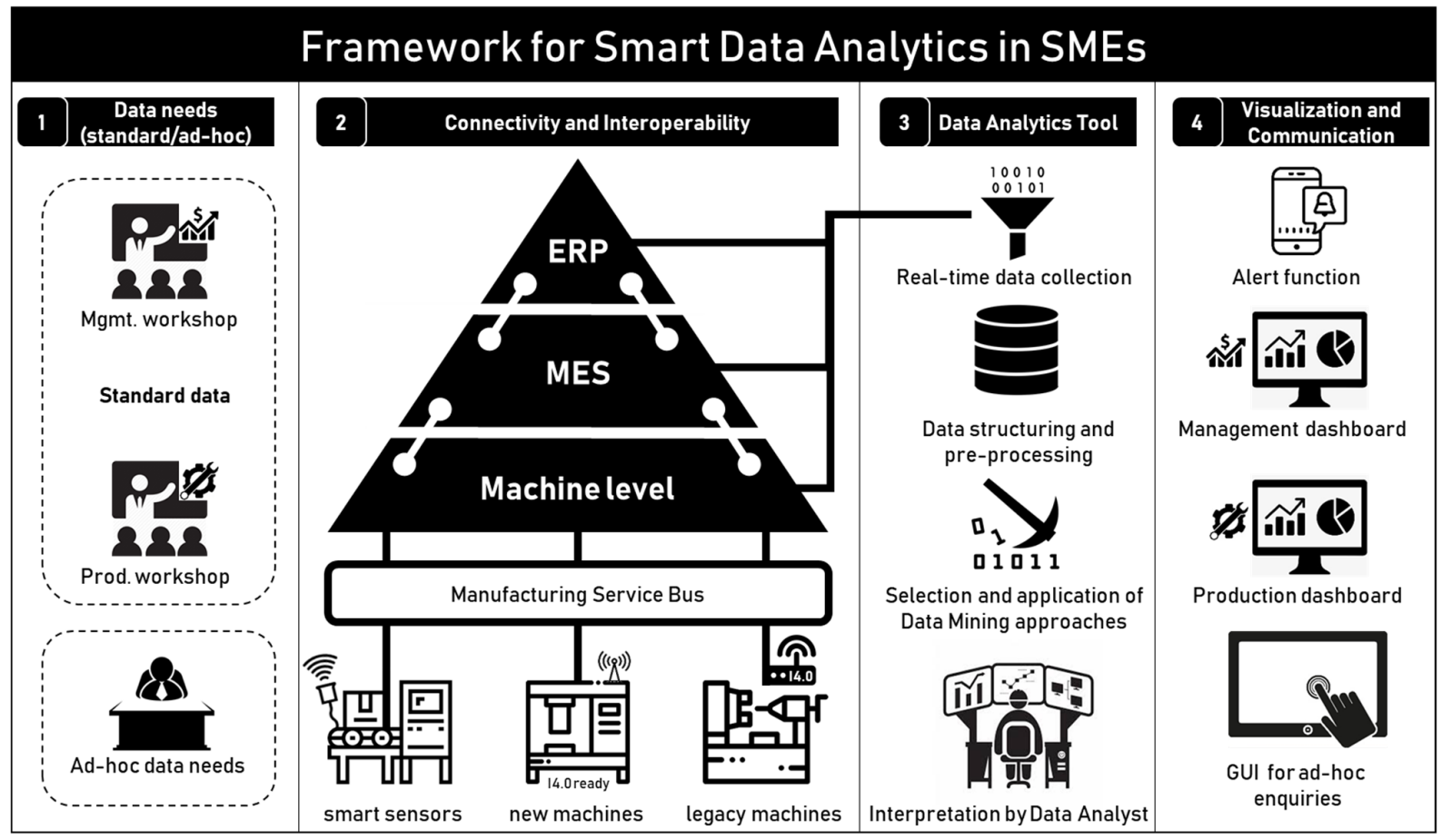

Fig. 2. Proposed framework for implementing smart data analytics in SME manufacturing.

\section{References}

1. Kagermann, H., Helbig, J., Hellinger, A., \& Wahlster, W. (2013). Recommendations for implementing the strategic initiative INDUSTRIE 4.0: Securing the future of German manufacturing industry; final report of the Industrie 4.0 Working Group. Forschungsunion, 2013.

2. AUDI, A. (2017). Dialoge smart factory. DialogeMagazin, 1, 10-15.

3. Büttner, K. H., \& Brück, U. (2017). Use Case Industrie 4.0-Fertigung im Siemens Elektronikwerk Amberg. In Handbuch Industrie 4.0 Bd. 4 (pp. 4570). Springer Vieweg, Berlin, Heidelberg.

4. Santos, C., Mehrsai, A., Barros, A. C., Araújo, M., \& Ares, E. (2017). Towards Industry 4.0: an overview of European strategic roadmaps. Procedia Manufacturing, 13, 972-979.

5. Li, L. (2018). China's manufacturing locus in 2025: With a comparison of "Made-in-China 2025" and "Industry 4.0". Technological Forecasting and Social Change, 135, 66-74.

6. Kraemer-Eis, H., \& Passaris, G. (2015). SME securitization in Europe. Journal of Structured Finance, 20(4), 97.

7. MISE (2016). Piano nazionale Impresa 4.0. https://www.mise.gov.it/index.php/it/industria40. Last update:update : 26. April 2019.

8. Li, B. H., Hou, B. C., Yu, W. T., Lu, X. B., \& Yang, C. W. (2017). Applications of artificial intelligence in intelligent manufacturing: a review. Frontiers of
Information Technology \& Electronic Engineering, 18(1), 86-96.

9. Al-Jarrah, O. Y., Yoo, P. D., Muhaidat, S., Karagiannidis, G. K., \& Taha, K. (2015). Efficient machine learning for big data: A review. Big Data Research, 2(3), 87-93.

10. Wang, J., Ma, Y., Zhang, L., Gao, R. X., \& Wu, D. (2018). Deep learning for smart manufacturing: Methods and applications. Journal of Manufacturing Systems, 48, 144-156.

11. Li, L., Su, F., Zhang, W., \& Mao, J. Y. (2018). Digital transformation by SME entrepreneurs: A capability perspective. Information Systems Journal, 28(6), 1129-1157.

12. Dittert, M., Härting, R. C., Reichstein, C., \& Bayer, C. (2017, June). A data analytics framework for business in small and medium-sized organizations. In International conference on intelligent decision technologies (pp. 169-181). Springer, Cham.

13. Llave, M. R. (2017). Business Intelligence and Analytics in Small and Medium-sized Enterprises: A Systematic Literature Review. Procedia Computer Science, 121, 194-205.

14. Babu, K. V. S. N. (2012). Business intelligence: Concepts, components, techniques and benefits. Components, Techniques and Benefits (September 22, 2012).

15. Pighin, M., \& Marzona, A. (2012, May). Data value in decision process: Survey on decision support system in small and medium enterprises. In 2012 Proceedings of the 35th International Convention MIPRO (pp. 1647-1654). IEEE.

\footnotetext{
*Corresponding author: andrewv@,wpi.edu
} 
16. Schuetz, C. G., Neumayr, B., Schrefl, M., \& Neuböck, T. (2016). Reference modeling for data analysis: The BIRD approach. International Journal of Cooperative Information Systems, 25(02), 1650006.

17. Schütz, C., \& Schrefl, M. (2014, September). Customization of domain-specific reference models for data warehouses. In 2014 IEEE 18th International Enterprise Distributed Object Computing Conference (pp. 61-70). IEEE.

18. Dyczkowski, M., Korczak, J., \& Dudycz, H. (2014, September). Multi-criteria evaluation of the intelligent dashboard for SME managers based on scorecard framework. In 2014 Federated Conference on Computer Science and Information Systems (pp. 1147-1155). IEEE.

19. Korczak, J., Dudycz, H., \& Dyczkowski, M. (2012, September). Intelligent dashboard for SME managers. Architecture and functions. In 2012 Federated Conference on Computer Science and Information Systems (FedCSIS) (pp. 1003-1007). IEEE.

20. Rao, J. J., \& Kumar, V. (2019). Technology Adoption in the SME Sector for Promoting Agile Manufacturing Practices. In Smart Intelligent Computing and Applications (pp. 659-665). Springer, Singapore.

21. Lee, J. Y., Yoon, J. S., \& Kim, B. H. (2017). A big data analytics platform for smart factories in small and medium-sized manufacturing enterprises: An empirical case study of a die casting factory. International Journal of Precision Engineering and Manufacturing, 18(10), 1353-1361.

22. Park, H., Ji, B., Lee, M., Choi, J., Lee, J., Bang, S. H., \& Cho, H. (2017, September). Conceptual Development Process of Mass-customizable Data Analytics Services for Manufacturing SMEs. In IFIP International Conference on Advances in Production Management Systems (pp. 194-201). Springer, Cham.

23. Soroka, A., Liu, Y., Han, L., \& Haleem, M. S. (2017). Big data driven customer insights for SMEs in redistributed manufacturing. Procedia CIRP, 63, 692-697.

24. Suh, H. P. (2001). Axiomatic Design: advances and applications MIT-Pappalardo series in Mechanical Engineering. Oxford University Press, USA.

25. Brown, C. A. (2005). Teaching axiomatic design to engineers-Theory, applications, and software. Journal of Manufacturing Systems, 24(3), 186-195.

26. Rojas, R. A., Rauch, E., Vidoni, R., \& Matt, D. T. (2017). Enabling connectivity of cyber-physical production systems: a conceptual framework. Procedia Manufacturing, 11, 822-829.

27. Dennert, A., Gössling, A., Krause, J., Wollschlaeger, M., \& Montoya, A. M. H. (2012, May). Vertical data integration in automation based on IEC 61499. In 2012 9th IEEE International Workshop on Factory Communication Systems (pp. 99-102). IEEE.
28. Schlechtendahl, J., Keinert, M., Kretschmer, F., Lechler, A., \& Verl, A. (2015). Making existing production systems Industry 4.0-ready. Production Engineering, 9(1), 143-148.

29. Menezes, S., Creado, S., \& Zhong, R. Y. (2017). Smart Manufacturing Execution Systems for Small and Medium-sized Enterprises. decision making, 13, 14.

30. Gronau, N., \& Lass, S. (2018). Digitalisierung der Produktion in KMU. In: Dominik T. Matt (ed.) KMU 4.0 Digitale Transformation in kleinen und mittelständischen Unternehmen, Berlin: Gito Verlag, 135-152.

31. Bosch (2019). Industry 4.0 retrofit project. https://www.bosch.com/stories/industry-4-0-retrofitproject/. Last update: 25. April 2019. 